\title{
Black-box Adversarial Attacks on Monocular Depth Estimation Using Evolutionary Multi-objective Optimization
}

\author{
Renya Daimo ${ }^{1}$,Satoshi $\mathrm{Ono}^{1}$,Takahiro Suzuki ${ }^{1}$ \\ ${ }^{1}$ Department of Information Science and Biomedical Engineering, Graduate School of \\ Science and Engineering, Kagoshima University \\ 1-21-40, Korimoto, Kagoshima, 890-0065 Japan
}

\begin{abstract}
This paper proposes an adversarial attack method to deep neural networks (DNNs) for monocular depth estimation, i.e., estimating the depth from a single image. Single image depth estimation has improved drastically in recent years due to the development of DNNs. However, vulnerabilities of DNNs for image classification have been revealed by adversarial attacks, and DNNs for monocular depth estimation could contain similar vulnerabilities. Therefore, research on vulnerabilities of DNNs for monocular depth estimation has spread rapidly, but many of them assume white-box conditions where inside information of DNNs is available, or are transferability-based blackbox attacks that require a substitute DNN model and a training dataset. Utilizing Evolutionary Multi-objective Optimization, the proposed method in this paper analyzes DNNs under the black-box condition where only output depth maps are available. In addition, the proposed method does not require a substitute DNN that has a similar architecture to the target DNN nor any knowledge about training data used to train the target model. Experimental results showed that the proposed method succeeded in attacking two DNN-based methods that were trained with indoor and outdoor scenes respectively.
\end{abstract}

\section{INTRODUCTION}

Monocular depth estimation, i.e. estimating the scene depth from a single image, has been widely studied because it is one of the fundamental prob- 
lems in computer vision $[1,2,3]$ and it includes various applications such as robotics, image refocusing, scene understanding, and so on $[2,4,5,6]$.

Recent advances in Deep Neural Networks (DNNs) have dramatically improved the performance $[7,8,9,10]$ and increased expectations for its applications to real-world problems.

On the other hand, some studies revealed that DNNs have a particular vulnerability in which an input image carefully perturbed by an attacker causes misrecognition [11]. Similar vulnerability is expected to exist in DNNs for monocular depth estimation, and recent studies about adversarial attack methods for DNNs for monocular depth estimation began to take place $[12,13]$. The above studies assume a white-box condition where internal information of DNNs can be used to generate adversarial examples, and are useful when developers of the DNNs verify the vulnerability by themselves. However, it is diffuicult for a third party to assess the security of the DNNs without permission to the internal information of the DNN. Since 2020, transferability-based black-box attack methods have been proposed $[14,15,16]$; however, the methods require a substitute DNN model with a similar architecture to a target DNN and a dataset that is also the same or similar to the one used to train the target DNN.

Therefore, this study proposes an adversarial attack method for verifying the vulnerability of DNNs for monocular depth estimation under a blackbox condition where the inside information of DNNs is not available. Unlike object recognition, DNNs for monocular depth estimation produce a depth map, i.e., estimated pixel-wise depth values, without confidential scores. The proposed method can generate adversarial examples only by referring to the depth maps because depth estimation is essentially a pixel-wise regression problem, whose output can be represented as continuous values.

To analyze the DNNs described above, the proposed method employs black-box optimization-based approach to design adversarial perturbations on textures of a target object. In particular, we adopt Evolutionary Multiobjective Optimization (EMO) that simultaneously minimizes the difference of depth values between the estimated depth map and a target map in addition to the perturbation amount.

The contribution of this paper summarized as follows:

- To the best of our knowledge, this is the first study about black-box optimization-based adversarial attacks that is not based on the transferability between DNN-based monocular depth estimation models. The 
proposed method can be applicable to proprietary services and systems.

- Black-box global optimization-based approach the proposed method employs does not require a substitute DNN to approximate the target DNN model, nor any information about training datasets the target model used.

- The proposed method adds perturbations only on the target object, so it can be developed into a physical attack. In addition, unlike the universal adversarial patch, the perturbation amount is moderate and varies depending on the target object, making it more difficult to defend.

- Two DNN models trained with indoor and outdoor scenes respectively have analyzed by the proposed method. By adding perturbations only to the target object texture, without the background or other objects, the proposed method produced adversarial examples where DNNs falsely predicted depth maps as if the target object had disappeared.

The rest of this paper organized as follows: Sec. 2 introduces some recent DNN-based monocular depth estimation models and representative adversarial attack methods. Sec. 3 describes the key ideas of the proposed method, the formulation of adversarial example generation as a multi-objective optimization problem, and the detailed algorithm of the proposed method. Sec. 4 demonstrates the effectiveness of the proposed method by analyzing two DNN-based monocular depth estimation models.

\section{RELATED WORK}

\subsection{Monocular depth estimation using DNNs}

Recent remarkable advances in DNNs have led to increasing studies on monocular depth estimation $[8,7,17,9,10]$. Eigen et al. proposed a method using a DNN consistng of two networks that perform global coarse-scare and local fine-scale prediction [7]. Laina et al. employed a fully convolutional network that enables higher resolution output while suppressing the number

of parameters to be trained [8]. Godard et al. adopted a self-supervised learning approach that achieved sufficient accuracy [17]. Hu et al. proposed a method of monocular depth estimation that produces a depth map with 
more accurate object boundaries by focusing multi-scale features and loss functions based on surface normals [10].

With the development of monocular depth estimation technology, research on its analysis is also being conducted. Dijk et al. showed that a model trained by in-vehicle camera scenes estimates an object depth by focusing on the vertical position of the object in an image while ignoring the image region size of the object, and that the model only partially recognizes changes of the pitch and roll angles of the camera [18]. Hu et al. clarified that DNN-based models select important edges independently from the edge strength and focuses not only on the boundary but the inside region of the objects [19]. They also showed that the regions around vanishing points are prioritized in the outdoor scenes.

\subsection{Adversarial attacks for $D N N$}

DNNs achieved state-of-the-art performance not only in monocular depth estimation but in various domains of computer vision [20, 21], speech recognition [22, 23], and so on; however, their vulnerability against adversarial perturbations has been clarified $[11,24,25]$. The early study by Goodfellow et al. proposed the Fast Gradient Sign Method (FGSM) to generate adversarial examples for image classification [11]. Carlini et al. proposed C\&W attack that formulates adversarial example generation as an optimization problem [26].

Some black-box attack methods have also been studied that does not require internal information such as gradients of loss functions. One main approach for black-box attack is transferability-based approach. Papernot et al. proposed a method to generate $\mathrm{AE}$ under $\mathrm{BB}$ conditions by constructing an alternative model of the target NN model [24]. Liu et al. proposed an ensemble-basd method to generate transferable adversarial examples [27]. Recently, boundary attack methods have been widely studied, which originated in Brendel et al. [28], which assumes hard-label black-box condition where only an assigned label is available.

The third approach, alongside the transferability-based approach and boundary attack, is black-box optimization-based approach, which originated in the study by Su et al. [29]. They proposed one pixel attack method using Differential Evolution [30] that modifies very few pixels to create adversarial examples. Suzuki et al. proposed a method using EMO that can generate robust adversarial examples against image conversion under black-box conditions [25]. Kuang et al. proposed a query efficient black-box optimization- 
based attack method using Covariance Matrix Adaptation Evolution Strategy [31]. The drawback of of this approach is the computational cost, which limits the scenarios where the attack methods are applicable; however, there are certain scenarios where this approach is useful. For instance, with the permission of the system developer, a large number of queries are allowed for the third parties to externally inspect the system for vulnerabilities. In particular, it is expected for the global black-box optimization-based approach to find unknown vulnerabilities different from ones found by transfer-based and boundary-based attacks.

Since 2019, research on adversarial attacks for monocular depth estimation has also begun. Yamanaka et al. showed that the depth of the target area is erroneously estimated by overwriting the local area of the input image [12]. Hu et al. attempted to analyze the mechanism of monocular depth estimation by generating adversarial examples [13]. Zhang et al. proposed a multi-task attack method based on transferability-based approach that can be applicable to black-box condition [14]. Mathew et al. also proposed a transferability-based black-box attack method generating adversarial patch and investigated cross-data transferability where the same DNN model with different two training datasets, assumed that one is opened and another is proprietary [15]. Wong et al. added perturbations on the whole area of the captured image to achieve target attacks [16]. They also tested a black-box attack using transferability between two models developed by the same researchers but reported that the perturbations optimized for one model does not transfer to another.

The above studies assumed white-box approach were difficult to apply to commercial services and systems, where the DNNs' internal information cannot be available, or performed transferability-based black-box attack that requires a DNN model with an architecture similar to the target model and a dataset similar to ones used to train the target model. In addition, no studies have conducted for techniques for adding perturbations only on the target object.

\section{The proposed black-box global optimization-based adversarial attack method}

\subsection{Key Ideas}

This paper proposes a black-box adversarial attack method against DNNs for monocular depth estimation. Followings are the key ideas of the proposed 
method.

\section{Idea 1: Employing Evolutionary Multi-objective Optimization (EMO) algorithm:}

EMO is a kind of black-box global optimization research field in evolutionary computation. Because EMO performs black-box global optimization without any prior to a target problem, unlike transferabilitybased approach, the proposed method does not require a substitute DNN that should have a similar architecture to a target DNN nor the dataset as ones used to train the target.

The main advantage of EMO is that it can simultaneously optimize more than one objective function such as performance versus cost [32]. Creating adversarial examples naturally involves multiple objective functions in trade-off relationship, such as accuracy versus visibility. A simple way to minimize the weighted linear sum of the functions can combine them into a single objective function; however, the weight parameters must be adjusted and the obtained solutions would be biased by the predetermined weights. Therefore, the proposed method formulates the problem as a multi-objective optimization and solves it using EMO. Because various objective funcions including non-differentiable, multimodal, and noisy ones can be employed, the proposed method is suitable for seeking unknown vulnerabilities in the monocular depth estimator.

\section{Idea 2: Dimension reduction technique based on block-wise per- turbation patterns is introduced:}

Naive formulation of adversarial example generation enlarges the problem size. Therefore this paper introduces block-wise perturbation generation, in which several small perturbation patterns are created while assigning appropriate perturbation patterns for all image blocks.

\section{Idea 3: Add perturbations to textures of a target object:}

Instead of adding perturbations to the entire region of a captured image, the proposed method perturbs textures of the target object. This is because, as discussed in the previous work [19], textures of object surfaces play an important role in monocular depth estimation in addition to its edges. Furthermore, by adding perturbations only to the 
object textures, the proposed method can be applicable to physical adversarial attacks $[33,34]$ in future.

\subsection{Formulation}

The proposed method introduces block-wise perturbation patterns, where assignments to image blocks of target object textures are $N_{\text {pat }} \times N_{\text {pat }}$ pixelblock perturbation patterns and their simultaneously optimized. The above scheme allows reducing the number of variables even when high-resolution textures are available.

Fig.1 illustrates the block-wise perturbation patterns. There are two types of design variables comprising a solution candidate $\chi$ as follows:

$$
\begin{aligned}
\chi & =\boldsymbol{x}^{(m a p)} \cup\left\{\boldsymbol{x}_{r}^{(p a t)}\right\}_{r \in\left\{1, \ldots, N_{A P}\right\}} \\
\boldsymbol{x}^{(m a p)} & =\left\{x_{u, v}^{(m a p)}\right\}_{(u, v) \in \boldsymbol{I}} \\
\boldsymbol{x}_{r}^{(p a t)} & =\left\{x_{p, q, r}^{(p a t)}\right\}_{p, q \in\left\{1, \ldots, N_{p a t}\right\}}
\end{aligned}
$$

Variable $x_{u, v}^{(\operatorname{map})}$ represents a pattern assignment map that block $(u, v)$ in the texture image $\boldsymbol{I}$, i.e., $x_{u, v}^{(\operatorname{map})} \in\left\{0,1,2, \ldots, N_{A P}\right\}$. If $x_{u, v}^{(\operatorname{map})}>0$ the corresponding block-wise perturbation pattern is applied to block $(u, v)$, otherwise, no perturbation is added to the block. Variable $x_{p, q, r}^{(p a t)}$ represents $r$-th block-wise perturbation pattern, that is, variation amount of intensity of pixel $(u, v)$ in the local coordinate of pattern $r\left(r \in\left\{1, \ldots, N_{A P}\right\}\right)$.

The proposed method simultaneously minimizes two objective functions: the depth estimation error and the perturbation amount. The proposed method mainly focuses on a target attack, that is, given a target depth map, the proposed method generated an adversarial example such that a target DNN estimates a depth map as close as possible to the given depth map.

The first objective function $f_{1}$ is the sum of absolute error between the target and estimated depth values.

$$
\operatorname{minimize} f_{1}(\boldsymbol{\chi})=\sum_{(w, h) \in \boldsymbol{I}}\left|d_{w, h}^{(\text {est })}(\boldsymbol{I}+\boldsymbol{\rho}(\boldsymbol{\chi}))-d_{w, h}^{(\text {target })}\right|
$$

where $d_{w, h}^{(\text {target })}$ and $d_{w, h}^{(e s t)}$ denotes the target and estimated depth values at pixel $(w, h) \in \mathcal{R}$, respectively, and $\mathcal{R}$ denotes the image region of the target object. The second objective function $f_{2}$ is the L2 norm of the perturbation $\boldsymbol{\rho}(\boldsymbol{\chi})$ generated by solution $\chi$

$$
\operatorname{minimize} f_{2}(\boldsymbol{\chi})=\|\boldsymbol{\rho}(\boldsymbol{\chi})\|_{2}
$$




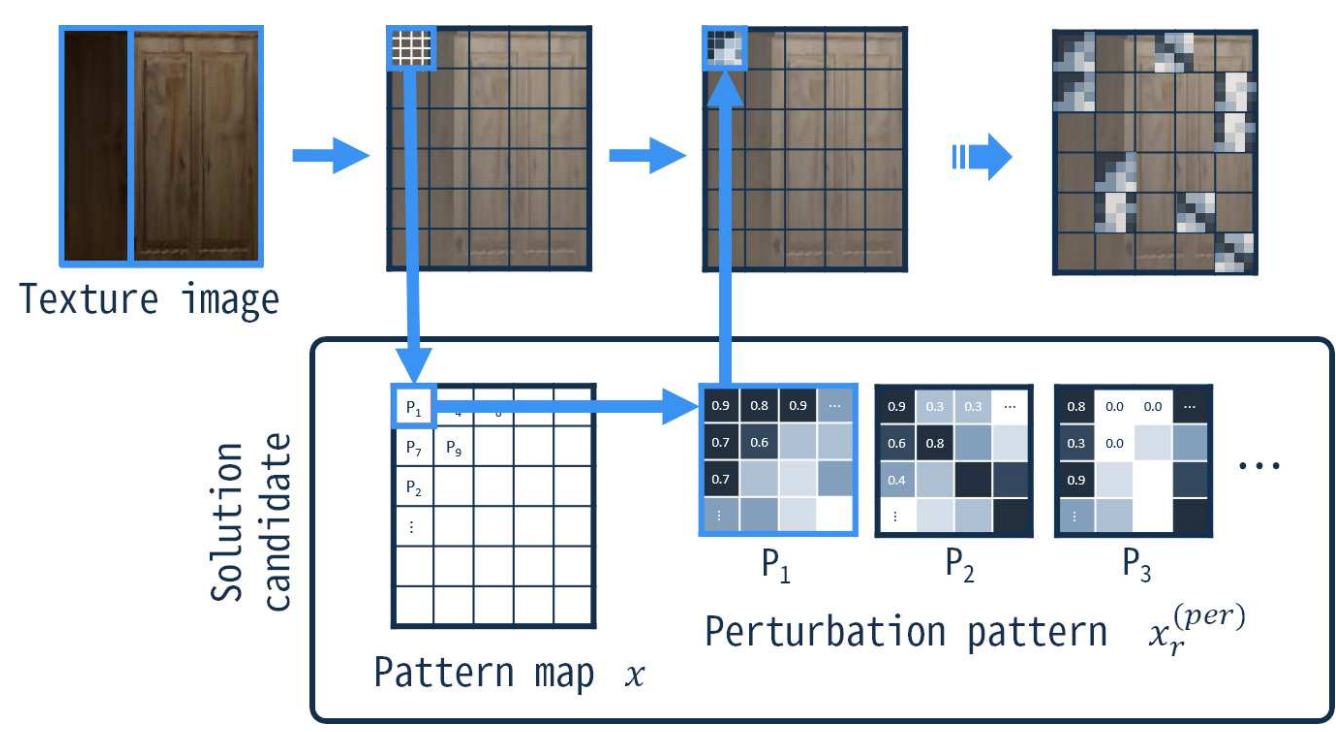

Figure 1: Block-wise perturbation patterns.

\subsection{Process flow}

The proposed algorithm can adopt any evolutionary multi-objective optimization algorithms such as NSGA-II[35] and MOEA/D[36]. In this study we employ MOEA/D to simultaneously optimize the two objective functions described in Sec. 3.2.

MOEA/D converts the approximation problem of the true Pareto Front into a set of single-objective optimization problems, for instance:

$$
\begin{aligned}
\operatorname{minimize} & g\left(\boldsymbol{x} \mid \boldsymbol{\lambda}^{j}, z^{*}\right)=\max _{i \in\left\{1, \ldots, N_{f}\right\}}\left\{\lambda_{i}^{j} \mid f_{i}(\boldsymbol{x})-z_{i}^{*}\right\} \\
\text { subject to } & \boldsymbol{x} \in \mathcal{F}
\end{aligned}
$$

where $\boldsymbol{\lambda}^{j}=\left(\lambda_{1}^{j}, \ldots, \lambda_{N_{f}}^{j}\right)$ are weight vectors $\left(\lambda_{i}^{j} \geq 0\right)$ and $\sum_{i=1}^{N_{f}} \lambda_{i}^{j}=1$, and $\boldsymbol{z}^{*}$ is a reference point in the objective function space calculated as follows:

$$
z_{i}^{*}=\min \left\{f_{i}(\boldsymbol{x}) \mid x \in \mathcal{F}\right\}
$$

By preparing $N_{D}$ weight vectors and optimizing $N_{D}$ scalar objective functions, MOEA/D finds various non-dominated solutions at one optimization.

At the beginning, MOEA/D determines the neighborhood relations for each weight vector $\boldsymbol{\lambda}_{i}$ by calculating the Euclidean distance between them so that $N_{n}$ neighboring weight vectors $\left\{\lambda^{k}\right\}$ are selected. Then MOEA/D 


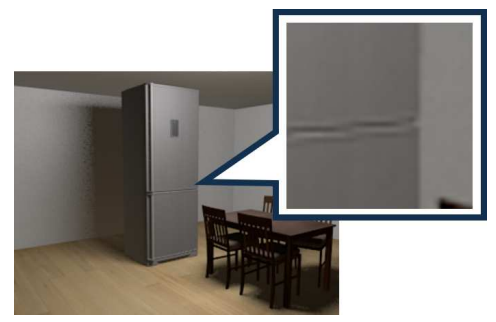

(a) Original image

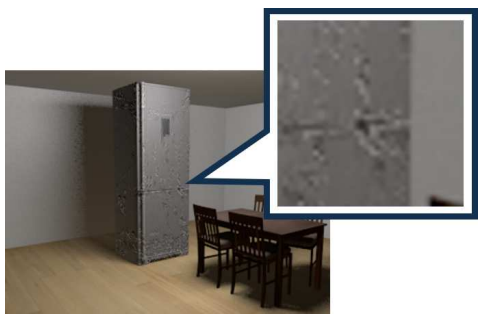

(b) Adversarial example

Figure 2: Example of an adversarial perturbation generated by the proposed method.

generates an initial population of solution candidates $\chi_{1}, \ldots \chi_{N_{p}}$ by sampling at uniform random from $\mathcal{F}$. Then, the initial reference point is calculated by eq. (7).

After initialization, MOEA/D repeats the processes of selection and population update including solution generation, evaluation, and solution update. In the selection process, $N_{f}$ best individuals are selected for $N_{f}$ objective functions respectively, and then, indexes of the subproblems are selected by tournament selection. Then the population is updated by applying crossover and mutation operators for each selected subproblem $i$. Before applying the operators, mating and update range is selected. That is, with the probability $\delta$, the update range $\mathcal{P}$ is limited to the neighborhood of $i$, otherwise $\mathcal{P}=1, \ldots, N_{d}$. Then, crossover is performed to randomly selected two indices $r_{1}$ and $r_{2}$, and generates a solution $\boldsymbol{\psi}$ by the same way as Differential Evolution (DE) [30]. That is, the component $\psi_{k}$ of $\boldsymbol{\psi}$ is calculated as follows:

$$
\bar{y}_{k}= \begin{cases}\chi_{k}^{i}+F\left(\chi_{k}^{r_{1}}-\chi_{k}^{r_{2}}\right) & \text { with probability } C R \\ \chi_{k}^{i} & \text { with probability } 1-C R\end{cases}
$$

where $F$ and $C R$ are control parameters in DE. Following the crossover, polynomial mutation [37] is applied to $\psi_{k}$ with the probability $p_{m}$ as follows:

$$
\begin{aligned}
\psi_{k} & =\bar{\psi}_{k}+\bar{\delta} \Delta_{\max } \\
\bar{\delta} & = \begin{cases}(2 u)^{\frac{1}{n+1}}-1 & \text { if } u<0.5 \\
1-[2(1-u)]^{\frac{1}{n+1}} & \text { otherwise }\end{cases}
\end{aligned}
$$

where $\Delta_{\max }$ represents the maximum permissible perturbance in the parent value $\bar{\psi}_{k}$, and $u$ is a random number in $[0,1]$. 
Table 1: DNN models analyzed in this study.

\begin{tabular}{l|l|l|c}
\hline Experiment & Model & data set & $\begin{array}{c}\text { input / output } \\
\text { image size [px] }\end{array}$ \\
\hline 1 & Full convolution architecture [8] & NYU Depth v2 & $\begin{array}{c}228 \times 304 / \\
160 \times 124\end{array}$ \\
\hline 2 & Self-Supervised Training [17] & KITTI & $\begin{array}{c}640 \times 192 / \\
640 \times 192\end{array}$ \\
\hline
\end{tabular}

Then the generated solution $\boldsymbol{\psi}$ is evaluated. The perturbation pattern $\boldsymbol{\rho}$ is generated and the target DNN model is applied to the perturbed image $\boldsymbol{I}+\boldsymbol{\rho}$. From the obtained depth map, values of $f_{1}(\boldsymbol{\chi})$ and $f_{2}(\boldsymbol{\chi})$ are calculated. From the evaluation results, the component $z_{j}$ of the reference point is replaced with $f_{j}(\boldsymbol{\psi})$ if $z_{j}>f_{j}(\boldsymbol{\chi})$ for each $j \in\left\{1, \ldots, N_{f}\right\}$. At the end of the main loop, the population is updated by the following procedure:

(1) Set $c=0$.

(2) If $c=n_{r}$ or $\mathcal{P}$ is empty, then go to (4). Otherwise, pick an index $k$ from $\mathcal{P}$ at random.

(3) If any of the following conditions are satisfied, then replace $\chi^{k}$ with $\boldsymbol{\psi}$ and set $c=c+1$.

$$
\begin{gathered}
\boldsymbol{\psi} \notin \mathcal{F} \wedge \chi^{k} \notin \mathcal{F} \wedge \operatorname{vio}(\boldsymbol{\psi})<\operatorname{vio}\left(\chi^{k}\right) \\
\boldsymbol{\psi} \in \mathcal{F} \wedge \chi^{k} \notin \mathcal{F} \\
\boldsymbol{\psi} \in \mathcal{F} \wedge \chi^{k} \in \mathcal{F} \wedge g\left(\boldsymbol{\psi} \mid \lambda^{k}, \boldsymbol{z}\right) \leq g\left(\chi^{k} \mid \lambda^{k}, \boldsymbol{z}\right)
\end{gathered}
$$

where $\operatorname{vio}(\cdot)$ denotes the amount of constraint violations.

(4) Remove $k$ from $\mathcal{P}$ and go back to (2).

\section{Evaluation}

In order to verify the effectiveness of the proposed method, we analyzed vulnerabilities of two DNN models trained with indoor and outdoor scenes respectively (Table 1) by generating adversarial examples. The target depth maps are designed as if target objects were removed from the scenes. First, the DNN model trained with indoor scenes was analyzed while changing 
target object type (Experiment 1-a), and the presence of objects other than the target (Experiment 1-b) by creating scenes using CG. Then, the real images of the actual scenes were tested (Experiment 1-c). Finally, another DNN model trained with outdoor scenes was analyzed (Experiment 2), and the proposed method was compared with the previous method (Experiment $3)$.

In this study, the proposed method was configured as follows: the Chebyshev method was selected as the scalarization function, the neighborhood size $N_{n}$ and the number of objective functions $N_{f}$ were set to 10 and 2, respectively, and, $\delta=0.8$, and $n_{r}=1$. The number of solution candidates $N_{p}$ and the limit of the number of generations were set to 100 and 5,000, respectively. The number of block-wise perturbation patterns $N_{A P}$ and its size $N_{\text {pat }}$ were set to 10 and $8 \times 8$, respectively.

\subsection{Experiment 1: analyzing DNN trained with indoor scenes 4.1.1. Experimental setup}

In Experiment 1, we analyzed Laina's method [8] trained using the indoor scene dataset NYU Depth v2. We employed the trained model ${ }^{1}$, and did not train the model by ourselves. In Experiments 1-a and 1-b, to control the components comprising indoor scenes, such as, walls, ceilings and floors of the room, and furniture items, CG images were rendered to create simple scenes containing the floor, walls, ceiling, and furniture items. Each target object was approximated as a rectangular shape, and perturbations were added to its textures of the two surfaces (front and side) obtained by projective transformation. Fig.2(a) shows an example scene consisting of one target object (a refrigerator) and other objects (a dining table and chairs), and Fig. 2(b) shows generated adversarial examples.

In order to quantify the effect of generated adversarial examples on depth estimation errors, we focused on a pseudo volume of a perturbed object that is defined as the sum of differences between the estimated depth of the front side of the target object and a given target depth $d_{(i, j)}^{(\text {target })}$ for pixels in the target object region $\mathcal{R}$ as follows:

$$
V=\Sigma_{(i, j) \in \mathcal{R}}\left|d_{(i, j)}^{(e s t)}-d_{(i, j)}^{(\text {target })}\right|,
$$

\footnotetext{
${ }^{1}$ https://github.com/iro-cp/FCRN-DepthPrediction
} 
which is essentially the same as $f_{1}$ but the region of interest is different.

\subsubsection{Experiment 1-a: Influence of target object shape}

In order to investigate the influence of the shape of the target object, we created scenes that have different objects such as a wardrobe, a refrigerator, a sofa, and a dining table set, while the viewpoint and the light source were

fixted. In scene $S_{5}^{(i n)}$, perturbation was added only to the top side of the dining tables set, whereas perturbation was added to two sides $\left(S_{1}^{(i n)}, \ldots, S_{3}^{(i n)}\right)$ or three sides $\left(S_{4}^{(i n)}\right)$.

From the results shown in Fig. 3, in scenes $S_{1}^{(i n)}$ to $S_{3}^{(i n)}$, the proposed adversarial examples succeeded in having the target DNN make false depth estimations as if the target object disappeared. In $S_{4}^{(i n)}$, the sofa seems to have moved backward as well although it is difficult to see at a first glance. However, it was difficult to fool the DNN in scene $S_{5}^{(i n)}$ by adding the perturbation only on the top size of the table set, which contains many rod-like shapes at the bottom.

\subsubsection{Experiment 1-b: Influence of the presence other object than the target}

Next, we investigated the influence of the presence of objects other than the target object in the scene. We created scenes $S_{6}^{(i n)}, S_{7}^{(i n)}, S_{8}^{(i n)}$ by putting a dining table set on scene $S_{1}^{(i n)}, S_{3}^{(i n)}, S_{4}^{(i n)}$, respectively.

Fig. 4 shows the results. The depth values of the target objects were changed without being affected by the table set.

\subsubsection{Experiment 1-c: Experiments on real images}

In addition to scenes rendered using CG in Experiments 1-a and 1-b, scenes $S_{9}^{(i n)}, S_{10}^{(i n)}$, and $S_{11}^{(i n)}$ that were real images selected from the training dataset were tested in this experiment. The target objects were a table shelf, a door, and a sofa on scenes $S_{9}^{(i n)}, S_{10}^{(i n)}$, and $S_{11}^{(i n)}$, respectively.

Fig. 5 shows the results. In scene $S_{9}^{(i n)}$, by adding perturbations, the DNN incorrectly recognized the scene as if there were no table shelf. Similarly, in scene $S_{10}^{(i n)}$, the depth estimation result of the generated adversarial example was as if the door did not exist. On the contrary, in scene $S_{11}^{(i n)}$, it was difficult to generate an adversarial example that fools the DNN. 


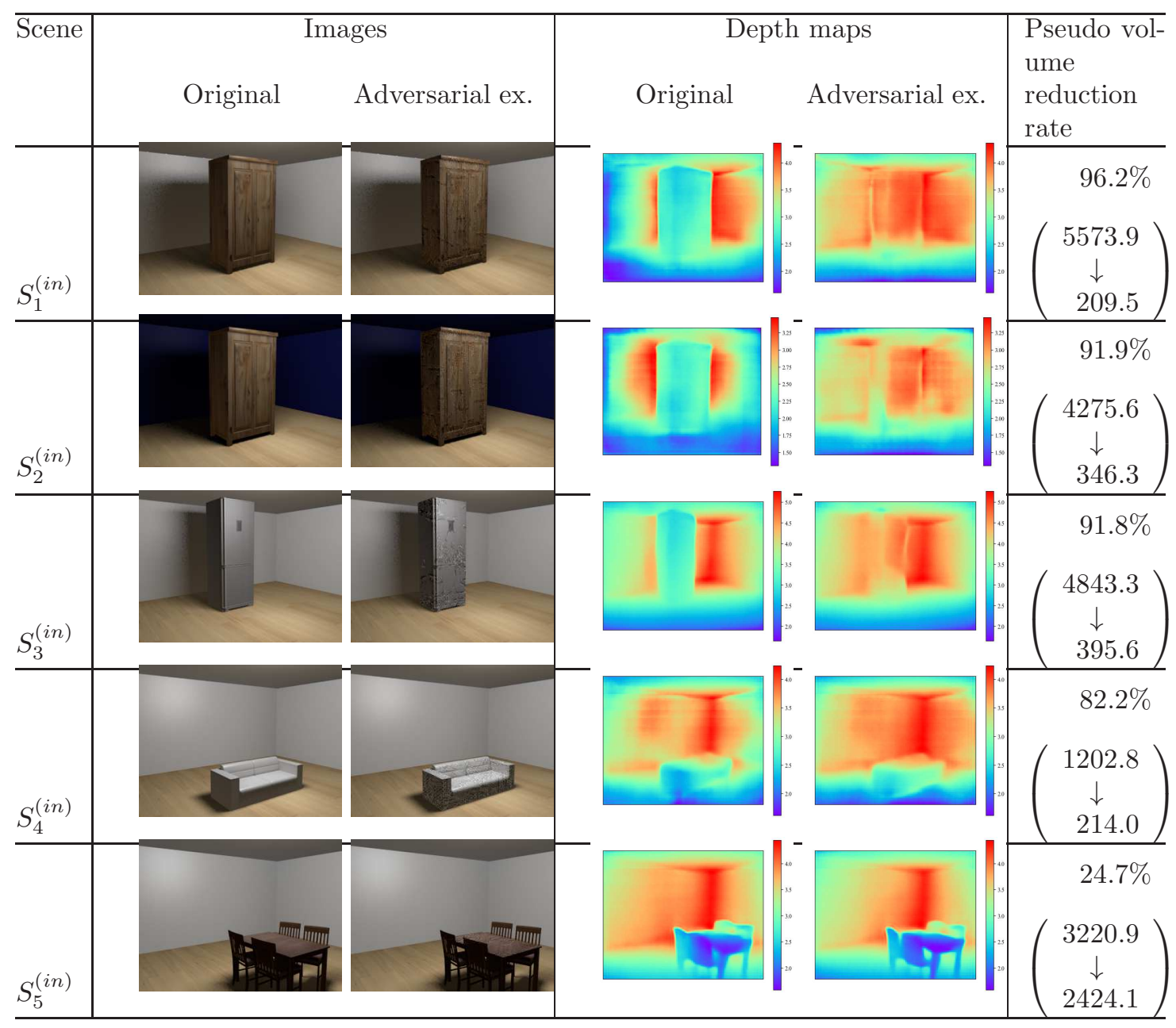

Figure 3: Effect of target object shape (Experiment 1-a)

The results in Experiment 1-a through 1-c suggested that perturbation on rectangular objects with height is likely to cause erroneous estimation.

\subsection{Experiment 2: Analysis of a model trained with outdoor scenes}

The second target DNN in our experiment is the model proposed by Godard et al. [17] trained on the outdoor scene dataset KITTI. Similar to 


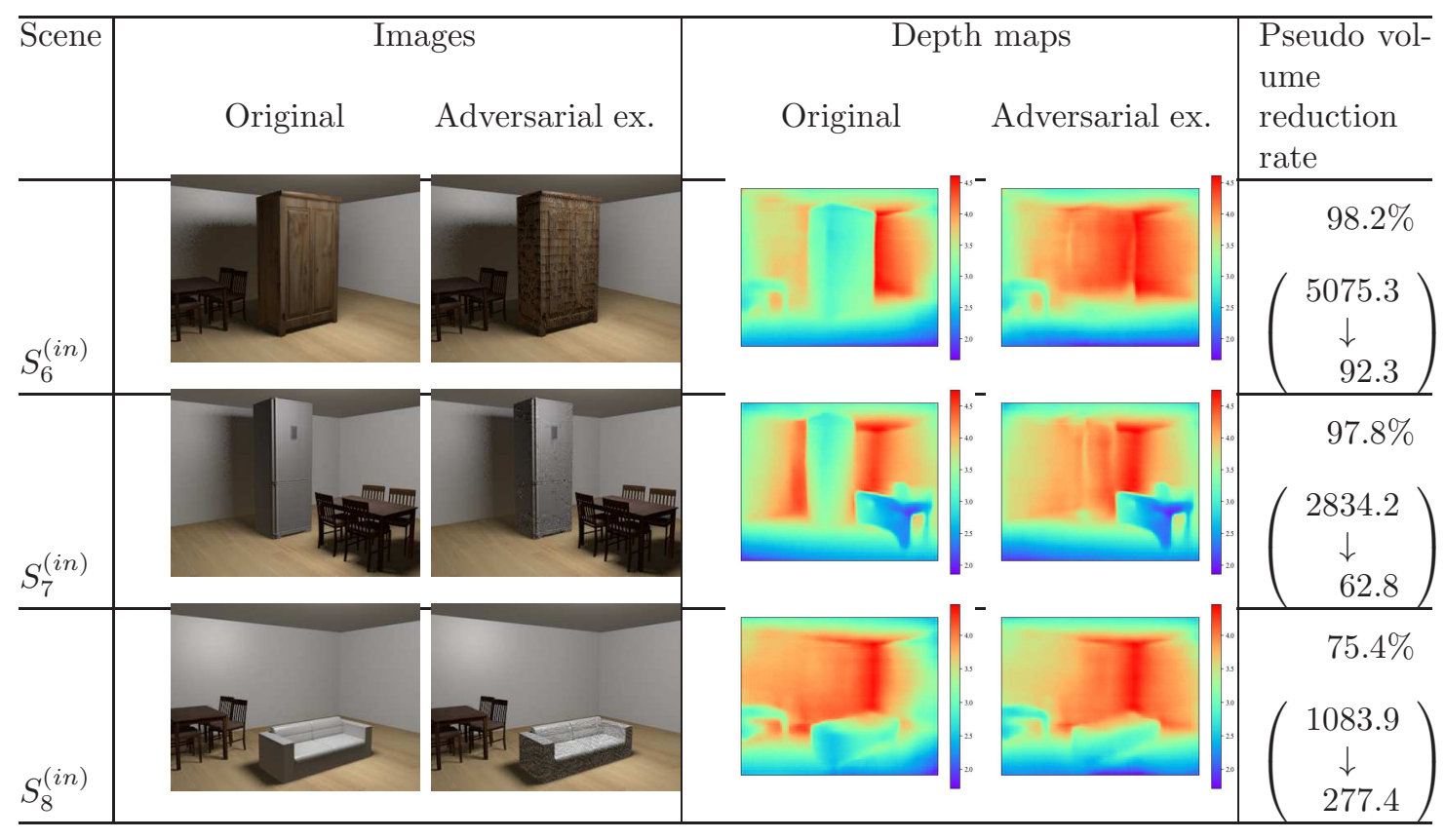

Figure 4: Effect of increasing number of objects (Experiment 1-b)

Experiment 1, we employed the trained model ${ }^{2}$ and did not trained the model by ourselves. The tested scenes $S_{1}^{(o u t)}, S_{2}^{(\text {out })}$, and $S_{3}^{(\text {out })}$ in this experiment were selected from the training data of the dataset.

Fig. 6 shows the results. Scenes $S_{1}^{(\text {out })}$ and $S_{2}^{(\text {out })}$ were misestimated as scenes where the target objects did not exist by adding perturbations, while the depth of the target object of $S_{3}^{(\text {out })}$ was not changed. In detail, in $S_{1}^{(\text {out })}$, the depth was estimated such that the top and middle of the target car disappeared, leaving only the bottom of the vehicle at about the same height as the sidewalk. Similarly, in $S_{2}^{(\text {out })}$, the depth was misestimated such that the hood and cabin disappeared, leaving only the front bumper. On the other hand, in scene $S_{3}^{\text {(out) }}$, there was little change in the depth of the target vehicle.

\footnotetext{
${ }^{2}$ https://github.com/nianticlabs/monodepth2
} 


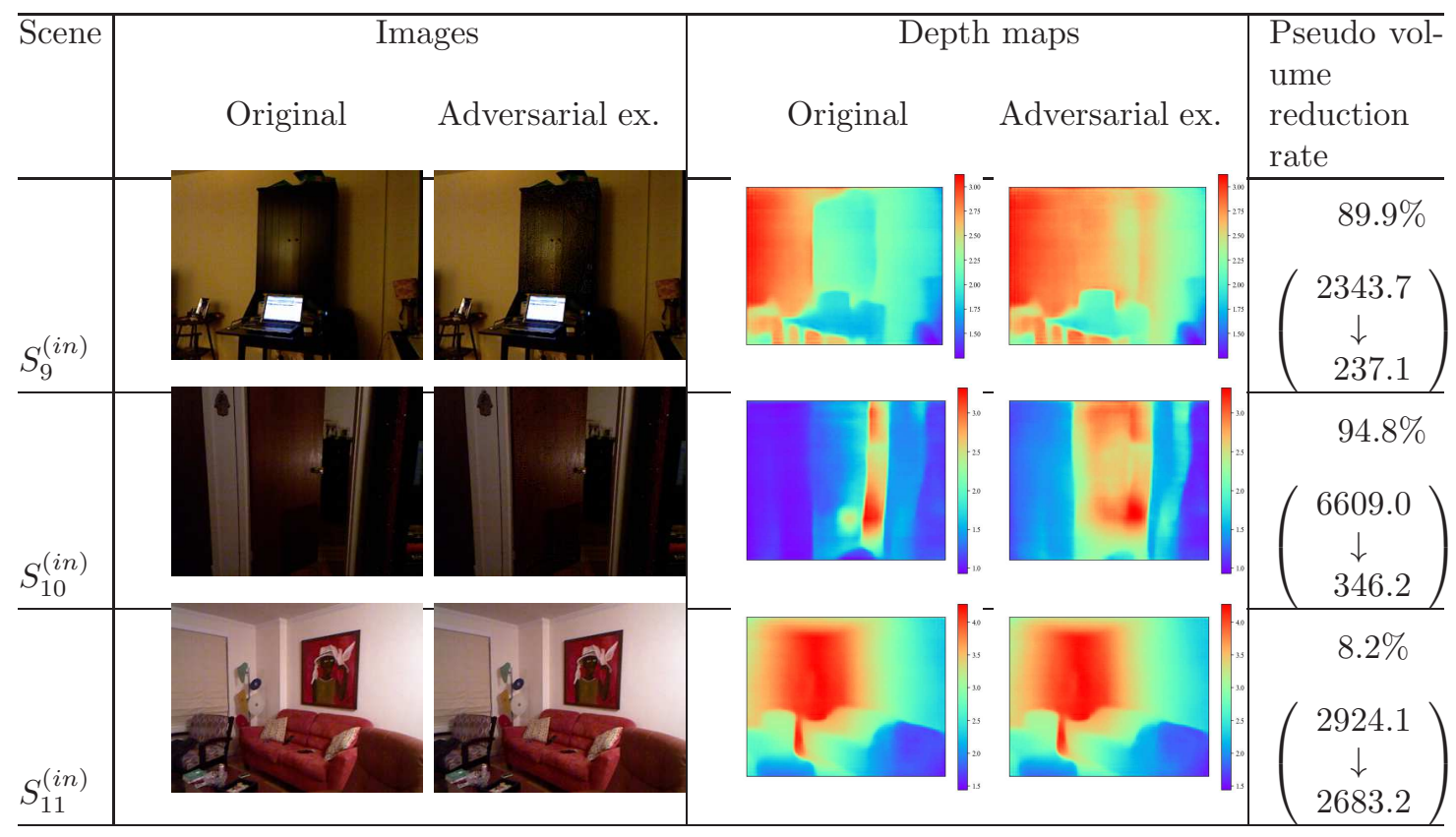

Figure 5: Experiment on real images (Experiment 1-d)

\subsection{Experiment 3: Comparison with previous work}

Finally, we compared our method with previous method [15], which produces universal adversarial patchs. This comparison was not fair in the following two points; first, the previous method designed the universal patch whereas the proposed method designed perturbations for each scene, and second, the previous method was white-box and untargeted attack whereas the proposed method was black-box and targeted attack. However, the two methods have in common that they apply perturbations only to target objects. In addition, most of other previous methods added perturbations to the entire scene images, making it difficult to develop them into physical attacks. For these reasons, this paper compared our method with Mathew's method.

The right two columns of Fig. 6 shows the results using two universal patches [15] designed for Godard's model [17]. The estimated disparity maps were not changed by the patches designed by the previous method in all the tested three scenes, whereas the perturbations by the proopsed method succeeded in misleading Godard's model in scene $S_{1}^{(\text {out })}$ and $S_{2}^{(\text {out })}$. 


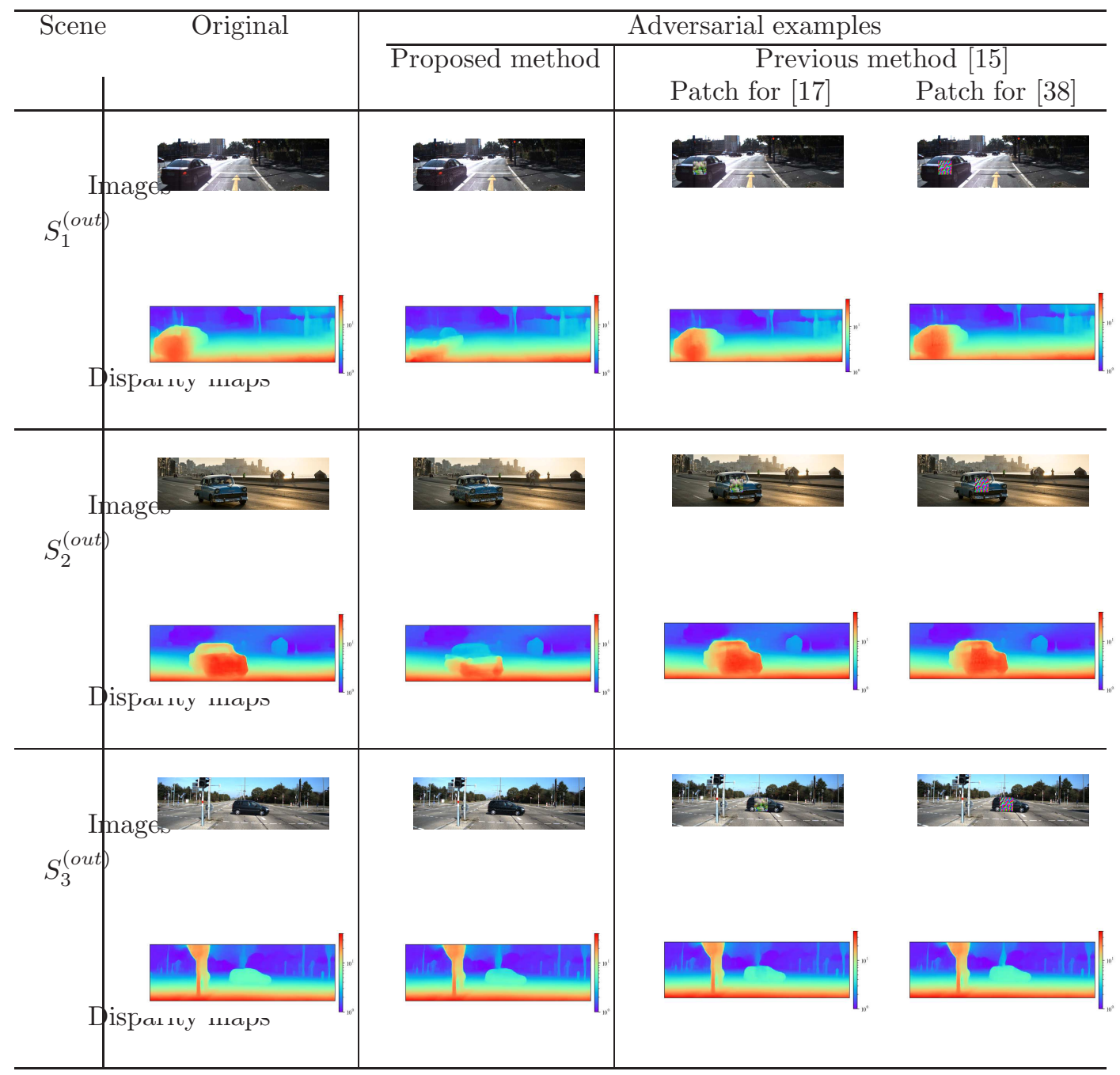

Figure 6: Results on Godard's model [17] in outdoor scenes (Experiment 2)

\section{Conclusion}

This paper proposed a method to generate adversarial examples that cause errors of monocular depth estimation under black-box conditions. The proposed method employs black-box optimization-based approach that does not require to have a substitute DNN and train it nor any information about training dataset. The proposed method successfully suppressed the number of design variables by introducing a block-wise perturbation scheme and by 
adding perturbation only on target object textures.

As demonstrated in the experiment, the proposed black-box attack method successfully changed the estimated depth of the target objects by adding perturbations only to the target, whereas previous studies added perturbations to the entire image $[13,14,16]$ or patches the compeletely overwrite the certain area of the scenes $[12,15]$. On the other hand, the experiments revealed the future task of this study where getting DNNs to misestimate the depth of rod-shaped objects such as dining chairs is more challenging.

At this stage, ths study has not considered reducing computational costs because we assumed the situation where DNN models are externally inspected with the permission of the developers to find vulnerabilities that cannot be found white-box attacks and transfer-based attacks. In future, we plan to speed up the proposed method by hybridizing it with boundary-based attack methods. 
[1] H. Laga, A survey on deep learning architectures for image-based depth reconstruction, arXiv preprint arXiv:1906.06113.

[2] A. Bhoi, Monocular depth estimation: A survey, arXiv preprint arXiv:1901.09402.

[3] T. Koch, L. Liebel, M. Körner, F. Fraundorfer, Comparison of monocular depth estimation methods using geometrically relevant metrics on the ibims-1 dataset, Computer Vision and Image Understanding 191 (2020) 102877. doi:https://doi.org/10.1016/j.cviu.2019.102877. URL http://www.sciencedirect.com/science/article/pii/ S1077314219301663

[4] J. Shi, X. Tao, L. Xu, J. Jia, Break ames room illusion: depth from general single images, ACM Transactions on Graphics (TOG) 34 (6) (2015) 1-11.

[5] K. [van Hecke], G. C. [de Croon], D. Hennes, T. P. Setterfield, A. Saenz-Otero, D. Izzo, Self-supervised learning as an enabling technology for future space exploration robots: Iss experiments on monocular distance learning, Acta Astronautica 140 (2017) 1 - 9 . doi:https://doi.org/10.1016/j.actaastro.2017.07.038. URL http://www.sciencedirect.com/science/article/pii/ S0094576517302862

[6] M. Mancini, G. Costante, P. Valigi, T. A. Ciarfuglia, J-mod 2: Joint monocular obstacle detection and depth estimation, IEEE Robotics and Automation Letters 3 (3) (2018) 1490-1497.

[7] D. Eigen, C. Puhrsch, R. Fergus, Depth map prediction from a single image using a multi-scale deep network, in: Advances in neural information processing systems, 2014, pp. 2366-2374.

[8] I. Laina, C. Rupprecht, V. Belagiannis, F. Tombari, N. Navab, Deeper depth prediction with fully convolutional residual networks, in: Int'l Conf. 3D vision, 2016, pp. 239-248.

[9] V. Casser, S. Pirk, R. Mahjourian, A. Angelova, Depth prediction without the sensors: Leveraging structure for unsupervised learning from monocular videos, in: AAAI Conf. Artificial Intelligence, Vol. 33, 2019, pp. 8001-8008. 
[10] J. Hu, M. Ozay, Y. Zhang, T. Okatani, Revisiting single image depth estimation: Toward higher resolution maps with accurate object boundaries, in: Winter Conf. Applications of Computer Vision, 2019, pp. $1043-1051$.

[11] I. J. Goodfellow, J. Shlens, C. Szegedy, Explaining and harnessing adversarial examples, arXiv preprint arXiv:1412.6572.

[12] K. Yamanaka, R. Matsumoto, K. Takahashi, S. Fujii, Adversarial examples for monocular depth estimation [in japanese], in: IPSJ SIG Tech. Rep. AVM, Vol. 2019-AVM-107, 2019.

[13] J. Hu, T. Okatani, Analysis of deep networks for monocular depth estimation through adversarial attacks with proposal of a defense method, arXiv preprint arXiv:1911.08790.

[14] Z. Zhang, X. Zhu, Y. Li, X. Chen, Y. Guo, Adversarial attacks on monocular depth estimation, arXiv preprint arXiv:2003.10315.

[15] A. Mathew, A. P. Patra, J. Mathew, Monocular depth estimators: Vulnerabilities and attacks, arXiv preprint arXiv:2005.14302.

[16] A. Wong, S. Cicek, S. Soatto, Targeted adversarial perturbations for monocular depth prediction, arXiv preprint arXiv:2006.08602.

[17] C. Godard, O. Mac Aodha, M. Firman, G. J. Brostow, Digging into selfsupervised monocular depth estimation, in: Proceedings of the IEEE international conference on computer vision, 2019, pp. 3828-3838.

[18] T. v. Dijk, G. d. Croon, How do neural networks see depth in single images?, in: Int'l Conf. Computer Vision, 2019, pp. 2183-2191.

[19] J. Hu, Y. Zhang, T. Okatani, Visualization of convolutional neural networks for monocular depth estimation, in: Int'l Conf. Computer Vision, 2019, pp. 3869-3878.

[20] L.-C. Chen, G. Papandreou, F. Schroff, H. Adam, Rethinking atrous convolution for semantic image segmentation, arXiv preprint arXiv:1706.05587. 
[21] J. Redmon, A. Farhadi, Yolo9000: better, faster, stronger, in: Proceedings of the IEEE conference on computer vision and pattern recognition, 2017, pp. 7263-7271.

[22] A. Hannun, C. Case, J. Casper, B. Catanzaro, G. Diamos, E. Elsen, R. Prenger, S. Satheesh, S. Sengupta, A. Coates, et al., Deep speech: Scaling up end-to-end speech recognition, arXiv preprint arXiv:1412.5567.

[23] D. Povey, A. Ghoshal, G. Boulianne, L. Burget, O. Glembek, N. Goel, M. Hannemann, P. Motlicek, Y. Qian, P. Schwarz, et al., The kaldi speech recognition toolkit, in: IEEE 2011 workshop on automatic speech recognition and understanding, no. CONF, IEEE Signal Processing Society, 2011.

[24] N. Papernot, P. McDaniel, S. Jha, M. Fredrikson, Z. B. Celik, A. Swami, The limitations of deep learning in adversarial settings, in: 2016 IEEE European symposium on security and privacy (EuroS\&P), IEEE, 2016, pp. $372-387$.

[25] T. Suzuki, S. Takeshita, S. Ono, Adversarial example generation using evolutionary multi-objective optimization, in: IEEE Congress on Evolutionary Computation, 2019, pp. 2136-2144.

[26] N. Carlini, D. Wagner, Towards evaluating the robustness of neural networks, in: 2017 ieee symposium on security and privacy (sp), IEEE, 2017, pp. 39-57.

[27] Y. Liu, X. Chen, C. Liu, D. Song, Delving into transferable adversarial examples and black-box attacks, arXiv preprint arXiv:1611.02770.

[28] W. Brendel, J. Rauber, M. Bethge, Decision-based adversarial attacks: Reliable attacks against black-box machine learning models, arXiv preprint arXiv:1712.04248.

[29] J. Su, D. V. Vargas, K. Sakurai, One pixel attack for fooling deep neural networks, CoRR abs/1710.08864. arXiv:1710.08864.

URL http://arxiv.org/abs/1710.08864

[30] R. Storn, K. Price, Differential evolution a simple and efficient heuristic for global optimization over continuous spaces, J. of Global Optimization 
11 (1997) 341-359.

URL http://dl .acm.org/citation. cfm?id=596061.596146

[31] X. Kuang, H. Liu, Y. Wang, Q. Zhang, Q. Zhang, J. Zheng, A cmaes-based adversarial attack on black-box deep neural networks, IEEE Access 7 (2019) 172938-172947.

[32] C. C. Coello, Evolutionary multi-objective optimization: a historical view of the field, IEEE computational intelligence magazine 1 (1) (2006) $28-36$.

[33] D. Song, K. Eykholt, I. Evtimov, E. Fernandes, B. Li, A. Rahmati, F. Tramer, A. Prakash, T. Kohno, Physical adversarial examples for object detectors, in: 12th \{USENIX\} Workshop on Offensive Technologies (\{WOOT $\}$ 18), 2018.

[34] K. Eykholt, I. Evtimov, E. Fernandes, B. Li, A. Rahmati, C. Xiao, A. Prakash, T. Kohno, D. Song, Robust physical-world attacks on deep learning visual classification, in: Proceedings of the IEEE Conference on Computer Vision and Pattern Recognition, 2018, pp. 1625-1634.

[35] K. Deb, A. Pratap, S. Agarwal, T. Meyarivan, A fast and elitist multiobjective genetic algorithm: Nsga-ii, Evolutionary Computation, IEEE Transactions on 6 (2) (2002) 182-197.

[36] Q. Zhang, H. Li, MOEA/D: A Multiobjective Evolutionary Algorithm Based on Decomposition, IEEE Transactions on Evolutionary Computation 11 (6) (2007) 712-731.

[37] K. Deb, M. Goyal, A combined genetic adaptive search (geneas) for engineering design, Computer Science and Informatics 26 (1996) 30-45.

[38] C. Godard, O. Mac Aodha, G. J. Brostow, Unsupervised monocular depth estimation with left-right consistency, in: Proceedings of the IEEE Conference on Computer Vision and Pattern Recognition, 2017, pp. 270279 . 